\title{
Control of the Collagen Fibril Diameter in the Equine Superficial Digital Flexor Tendon in Horses by Decorin
}

\author{
Takafumi WATANABE ${ }^{1)}$, Yoshinao HOSAKA ${ }^{1)}$, Etsuko YAMAMOTO ${ }^{1)}$, Hiromi UEDA ${ }^{1)}$, Kenji SUGAWARA ${ }^{2)}$, \\ Heishichiro TAKAHASHI ${ }^{2)}$ and Kazushige TAKEHANA ${ }^{1) *}$ \\ ${ }^{1)}$ Department of Veterinary Anatomy, School of Veterinary Medicine, Rakuno Gakuen University, Ebetsu, Hokkaido 069-8501 and \\ ${ }^{2)}$ Center for Advanced Research of Energy Technology, Sapporo, Hokkaido University, Hokkaido 060-8628, Japan
}

(Received 3 December 2004/Accepted 9 May 2005)

\begin{abstract}
The distribution pattern of collagen fibril diameter in the equine superficial digital flexor tendon (SDFT) is known to differ in central and peripheral areas of some regions. This study reports the essence of collagen fibril differences among different regions of the equine SDFT by transmission electron microscopic (TEM) and high-voltage electron microscopic observations and biochemical analysis. The distribution of large collagen fibrils increased but the density of collagen fibrils decreased from the proximal metacarpal region to the distal metacarpal region. Large collagen fibrils with an irregular cross-sectional profile were found more frequently in the middle metacarpal region than in other regions. Three-dimensional reconstruction of images of irregularly shaped collagen fibrils revealed that these fibrils are formed through fusion of small collagen fibrils with large ones. The amount of decorin, which reportedly inhibits the lateral fusion of collagen fibrils, decreased in the direction of the distal metacarpal region. On the other hand, the size of decorin gradually increased in the direction of the distal metacarpal region. These results suggest that regional differences in collagen fibril distribution and density of collagen fibrils in the SDFT are due, at least in part, to fusion of collagen fibrils and the concomitant regional differences in the amount and size of decorin.
\end{abstract}

KEY WORDS: assembly, collagen, decorin, equine, superficial digital flexor tendon.

J. Vet. Med. Sci. 67(9): 855-860, 2005

Connective tissues such as tendons and ligaments are primarily composed of collagen fibrils. It is generally accepted that heterotypic collagen fibrils are the main component of mature connective tissue. Tendons are known to be composed of collagen types I, III and V [7]. A hybrid form of fibrils formed from interaction between types I and V collagen using an in vitro self-assembly system has been shown to play a regulatory role in fibril diameter $[1,5]$. Many studies have suggested that interactions between collagens and proteoglycans and/or glycosaminoglycans (GAGs) may also play a role in the formation of collagen fibrils.

Decorin, a small leucine-rich proteoglycan and a dominant dermatan sulfate (DS), binds to the surfaces of collagen fibrils, and it has been suggested that fibril-associated decorin regulates collagen fibril assembly and lateral growth in many types of connective tissue such as tendon, skin, and cornea $[3,6,9,25]$. In addition, the interfibrillar space of collagen fibrils is probably determined by decorin DS [23, 24]. Molecular size of decorin DS varies under pathologic and developmental conditions in correlation with sizes of collagen fibrils $[10,15]$. Although variation in size of collagen fibrils in different conditions has been reported, the investigation of sizes of collagen fibrils throughout the whole tendon length has not been done. Our study suggested differences in diameters of collagen fibrils in various regions of the equine superficial digital flexor tendon (SDFT) (unpublished date). It was found that the mean

\footnotetext{
* Correspondence to: Takehana, K., Department of Veterinary Anatomy, School of Veterinary Medicine, Rakuno Gakuen University, Ebetsu, Hokkaido, 069-8501, Japan.
}

diameter of collagen fibrils in the equine SDFT is smallest in the proximal region and gradually increases in the direction of the distal region. However, the mechanism by which collagen fibril diameter changes has not been elucidated. Therefore, the present study was carried out to clarify the morphological and biochemical characteristics of collagen fibrils in different regions of the equine SDFT.

\section{MATERIALS AND METHODS}

Tissue samples obtained from normal SDFTs of one male and three female Thoroughbred horses between $2-3$ years of age were used. The horses were housed at the Department of Veterinary Pathology, Rakuno Gakuen University, Japan. After sedation with medetomidine hydrochloride $(16 \mathrm{mg} / \mathrm{kg}$, IM) and anesthetic induction with thiopental sodium $(6 \mathrm{mg} /$ $\mathrm{kg}$, IV), tissue samples were surgically removed. All experiment animals were approved by the Ethics Committee and the Institutional Animal Use and Care Committee at Rakuno Gakuen University prior to the experiment. Three pieces of 2-cm-long SDFT samples were excised from three different regions and named according to their adjacent bone: proximal metacarpal (pM), middle metacarpal $(\mathrm{mM})$ and distal metacarpal (dM) (Fig. 1-a). A sample was also obtained from the central zone of each region (Fig. 1-b). The samples in each region were hemisected: one was quickly frozen in liquid nitrogen and stored at $-80^{\circ} \mathrm{C}$ for biochemical analysis and the other was prepared for morphological observation.

Morphological observation: Each sample was carefully sliced under a dissecting microscope into $1 \times 1 \times 2 \mathrm{~mm}$ blocks. The samples were immersed in $3 \%$ glutaraldehyde 


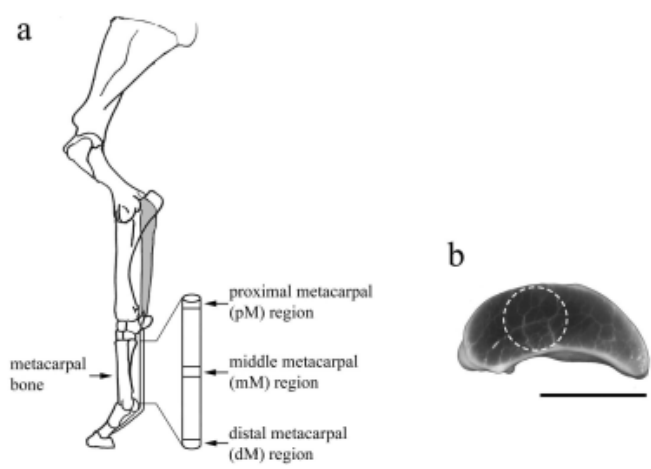

Fig. 1. a: Schema of the left forelimb and superficial digital flexor tendon (SDFT) and its muscle of a horse showing the three regions examined in this study. b: Cross-sectional image of the metacarpal region of SDFT. The dotted line indicates the central zone. The section was analyzed by morphological and biochemical methods. Bar $=10 \mathrm{~mm}$.

in $0.1 \mathrm{M}$ phosphate buffer, washed in phosphate buffer, postfixed in $1 \%(\mathrm{w} / \mathrm{v}) \mathrm{OsO}_{4}$ for $30 \mathrm{~min}$, dehydrated through a graded series of ethanol, and embedded in Quetol 812 (Nisshin EM, Tokyo, Japan). Ultrathin sections were obtained on an ultramicrotome (Reichert Supernova System; Leica, Vienna, Austria) and mounted on copper grids. The sections were stained with $0.2 \%(\mathrm{w} / \mathrm{v})$ tannic acid solution (Merck, Tokyo, Japan) for $15 \mathrm{~min}$, then with $1 \%(\mathrm{w} / \mathrm{v})$ uranyl acetate for $5 \mathrm{~min}$ and finally with $2 \%(\mathrm{w} / \mathrm{v})$ lead citrate for $5 \mathrm{~min}$. Tissue samples were examined under a transmission electron microscope (TEM: JEM-1220; JEOL, Tokyo, Japan) at an acceleration voltage of $80 \mathrm{kV}$. Collagen-to-noncollagen ratio in the extracellular matrix was characterized by the collagen fibril index (CFI) [8]. The total area for measuring CFI was $4 \mu \mathrm{m}^{2}$ in one sample. Though the variable in relative collagen density is always expected and a matter of fact that larger fibrils occupy a larger area than the smaller fibrils of the same number, direct measurement of collagen fibril diameter is probably not appropriate. Since determination of mass average diameter (MAD) could provide more functional information than determination of mean collagen fibril diameter, morphology of the collagen fibril population in this study was characterized by MAD according to the method of Flint et al. [11] instead of mean collagen fibril diameter. The MAD of each region was obtained by measuring diameters of 600 fibrils in one sample. In addition, thin sections of $500-700 \mathrm{~nm}$ in thickness were stained with $1 \%(\mathrm{w} / \mathrm{v})$ uranyl acetate for 60 min and 2\% (w/v) lead citrate for $15 \mathrm{~min}$ and were examined at various tilt angles with a high-voltage electron microscope (JEM ARM-1300; JEOL, Tokyo, Japan) at an acceleration voltage of $1,250 \mathrm{kV}$. Three-dimensional reconstruction was performed using TEM Tomograph software (JEOL, Tokyo, Japan).

Western Blot analysis: Samples, which were obtained from each tendon region, were homogenized and solubilized with CelLytic (Sigma, St. Louis, MO, U.S.A.) at $4^{\circ} \mathrm{C}$ according to the manufacturer's directions. Protein concentration was uniformalized by Lowry's method. To examine the molecular size and the amounts of decorin and DSdepleted core protein of decorin, samples were divided into 2 groups: One group was not treated and the other group was treated with chondroitinase ABC $(1 \mathrm{U} / \mathrm{m} l)(1: 1$; Seikagaku Kogyo, Tokyo, Japan) for $4 \mathrm{hr}$ at $37^{\circ} \mathrm{C}$ to digest dermatan sulfate. Then samples were boiled for 5 min with SDS sample buffer containing 2-mercapethanol and subjected to sodium dodecyl/polyacrylamide gel electrophoresis (SDS/ PAGE) $(10 \%$ gel) followed by Western blotting. Samples on gel were transferred to polyvinylidene fluoride (PVDF) membranes (Millipore Corporation, Billerica, MA, U.S.A.). Non-specific background was blocked in Blochace (Yukijirushi, Tokyo, Japan) for $1 \mathrm{hr}$. Each membrane was then washed four times with Tris-buffered saline ( $\mathrm{pH}$ 7.6) containing $0.2 \%$ Tween 20 (TBST). In order to examine decorin and its DS-depleted core protein, each membrane was incubated with an antibody against decorin using antihuman dermatan sulfate proteoglycan (1 in 10000 TBST; Seikagaku, Tokyo, Japan). After washing the blots four times with TBST, the bound antibodies were visualized with peroxidase-labeled anti-mouse IgG (Amersham Biosciences, Buckinghamshire, UK) for $1 \mathrm{hr}$ at room temperature and developed with an ECL plus Western Blotting Detection System (Amersham, UK) according to the manufacturer's instructions. The immunoreactivity of decorin and that of DS-depleted core protein of decorin were analyzed using the software NIH image, version 1.61.

Statistical analysis: Morphological analysis to determine frequency of collagen fibril distribution and density was performed on four sections from each region. Then data on fibril diameter and density of collagen obtained from independent experiments were combined. Mean differences between groups were tested with Scheffe test at $P<0.05$.

\section{RESULTS}

TEM images and histograms of collagen fibril diameters are shown in Fig. 2. Diameters of collagen fibrils in the pM, $\mathrm{mM}$ and $\mathrm{dM}$ regions were distributed with bimodal peaks at $20-40$ and $180-220 \mathrm{~nm}$. MAD values in the $\mathrm{pM}, \mathrm{mM}$ and $\mathrm{dM}$ regions were $132.8,145.5$ and $160.6 \mathrm{~nm}$, respectively. The ratio of collagen fibrils with large diameters $(>200 \mathrm{~nm})$ was lowest in the $\mathrm{pM}$ region and gradually increased in the direction of the $\mathrm{dM}$ region. Differences between mean diameters in the $\mathrm{pM}$ and $\mathrm{mM}$ regions and between mean diameters in the $\mathrm{pM}$ and $\mathrm{dM}$ regions were significant. Mean CFIs in the $\mathrm{pM}, \mathrm{mM}$ and $\mathrm{dM}$ regions were 73.6, 72.3 and $68.2 \%$, respectively, the value gradually decreasing in the direction of the $\mathrm{dM}$ region. Though comparison between $\mathrm{pM}$ and $\mathrm{dM}$ regions showed significant differences, there was no significant difference between two adjacent regions ( $\mathrm{pM}$ and $\mathrm{mM}$ regions and $\mathrm{mM}$ and $\mathrm{dM}$ regions). Moreover, MAD increased and CFI decreased toward the distal end of the metacarpus. 

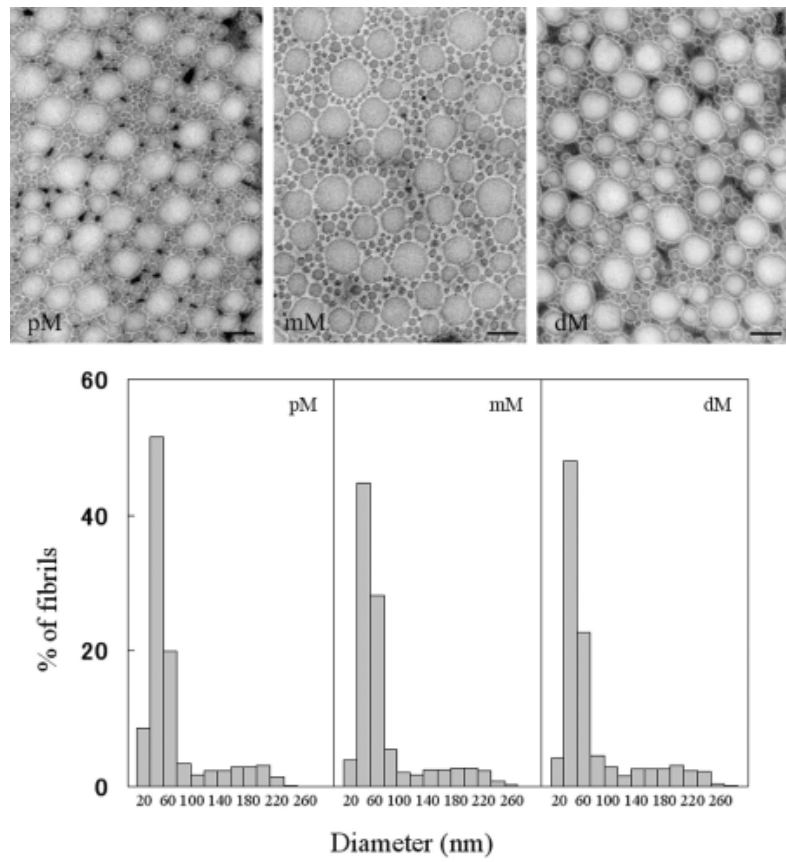

Fig. 2. Electron micrographs show cross sections of collagen fibrils in the proximal metacarpal $(\mathrm{pM})$ region, middle metacarpal $(\mathrm{mM})$ region and distal metacarpal $(\mathrm{dM})$ region of a SDFT. Small collagen fibrils are arranged between large fibrils in the three regions. Dark fibrils are thought to be elastic ones. Bar $=200 \mathrm{~nm}$. Histogram shows size of collagen fibril diameter. The distribution of collagen fibrils with large diameters increases from the $\mathrm{pM}$ region to the $\mathrm{dM}$ region. $\mathrm{N}=2,400$.
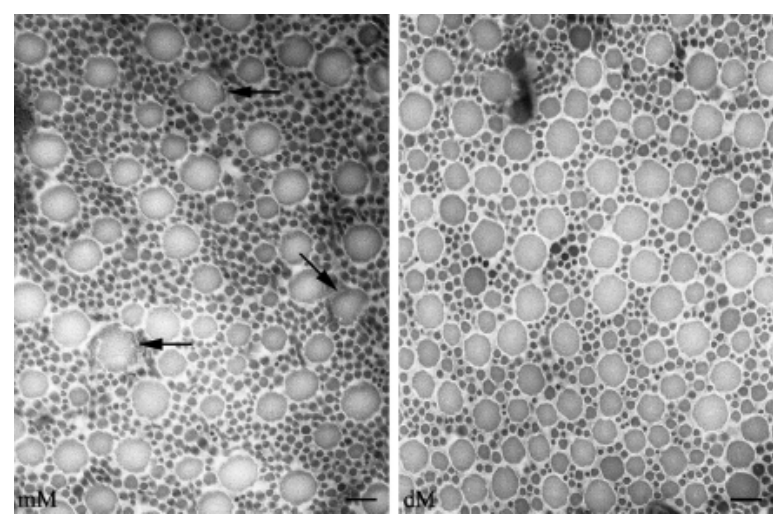

Fig. 3. High-voltage electron micrographs of 500-700-nm-thick cross sections of the $\mathrm{mM}$ and $\mathrm{dM}$ regions. Rugged collagen fibrils (arrows) were observed in the $\mathrm{mM}$ region but not in the $\mathrm{dM}$ region. $\mathrm{Bar}=200 \mathrm{~nm}$.

High-voltage electron microscopic observation: The cross-sectional shapes of most collagen fibrils were circular. Only a few collagen fibrils in the $\mathrm{mM}$ and $\mathrm{dM}$ regions with larger diameters than $200 \mathrm{~nm}$ had a rugged appearance. Rugged fibrils in the $\mathrm{mM}$ region were identified more frequently under a high-voltage electron microscope than
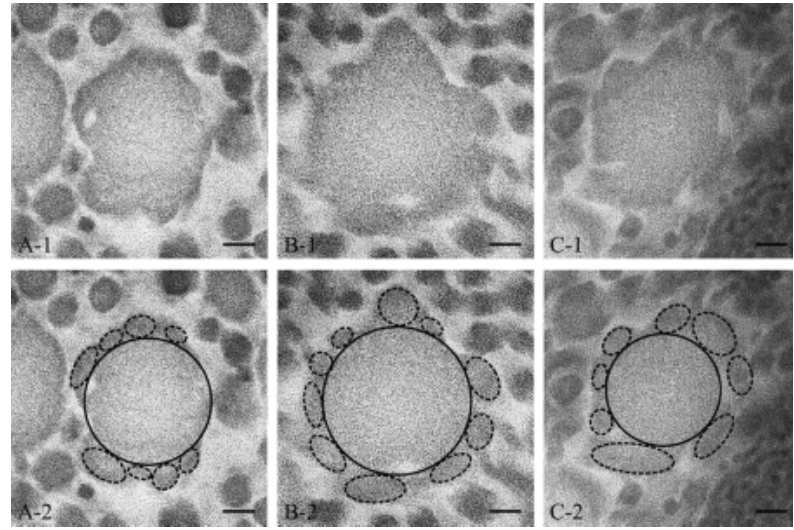

Fig. 4. High magnification of rugged collagen fibrils observed in high-voltage electron micrographs. Rugged collagen fibrils (A1, B-1, C-1) were separated into two groups, a large circular zone (line) and several small oval zones (dotted line) (A-2, B-2, $\mathrm{C}-2)$. Diameters of large fibrils are more than $200 \mathrm{~nm}$. The diameter of the minor length of the oval zone is less than $80 \mathrm{~nm}$. Bar $=50 \mathrm{~nm}$

under a transmission electron microscope (Fig. 3). However, identification of rugged fibrils in the $\mathrm{dM}$ region was not frequent. Rugged fibrils in the $\mathrm{mM}$ region appeared in two different patterns: 1) a large circular zone centering on the core of the rugged fibrils and 2) several small oval zones protruding from the large circular zone. The diameter of the large circular zone was approximately $200 \mathrm{~nm}$, and that of the oval zone (took the minor length to diameter) was less than $80 \mathrm{~nm}$ (Fig. 4).

High-voltage electron micrographs of rugged collagen fibrils obtained at various tilt angles were three-dimensionally reconstructed using computer-assisted reconstruction techniques (Fig. 5). The three-dimensionally reconstructed images showed that small collagen fibrils aggregated laterally to large collagen fibrils (Fig. 6).

Western blot analysis: Decorin (70-100 kDa) was detected in all three regions of tendon samples processed through Western blot analysis. However, molecular sizes of decorin in the upper regions were slightly smaller than those in the lower regions (Fig. 7-a). In addition, amount except molecular size of its core protein $(45 \mathrm{kDa})$ gradually decreased in a downward direction (Fig. 7-b).

\section{DISCUSSION}

By morphological and biochemical analyses, we obtained four novel findings: 1) the distribution of collagen fibril diameters in the equine SDFT varied from the $\mathrm{pM}$ to $\mathrm{dM}$ regions, 2) the assembly of collagen fibrils was often observed in the $\mathrm{mM}$ region, 3 ) the amount of decorin gradually decreased in the direction of the $\mathrm{dM}$ region and 4) only decorin DS increased in size from the $\mathrm{pM}$ to $\mathrm{dM}$ regions.

Cherdchutham et al. [8] reported that modal changes of collagen fibril diameter distribution in SDFTs of foals aged 2-11 months occur uniformly without regional preferences. 


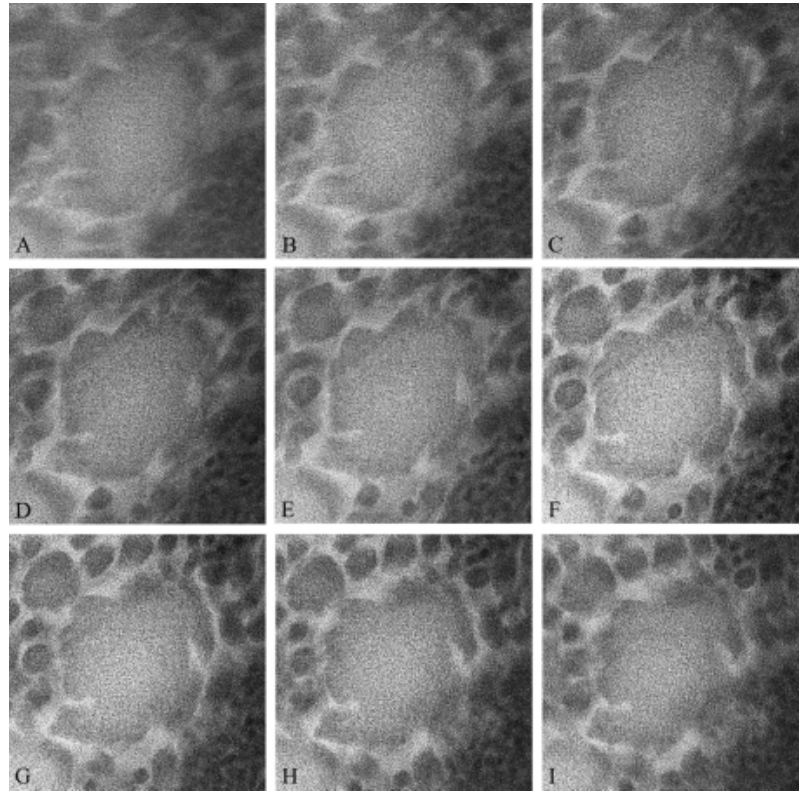

Fig. 5. High-voltage electron micrographs that Fig. 5-C was photographed at $-20^{\circ},-15^{\circ},-10^{\circ},-5^{\circ}, 0^{\circ},+5^{\circ},+10^{\circ},+15^{\circ}$ and $+20^{\circ}$ (A-I) from horizontal. The shape of A is nearly circlular, however it is observed to become the rugged shape gradually toward I.

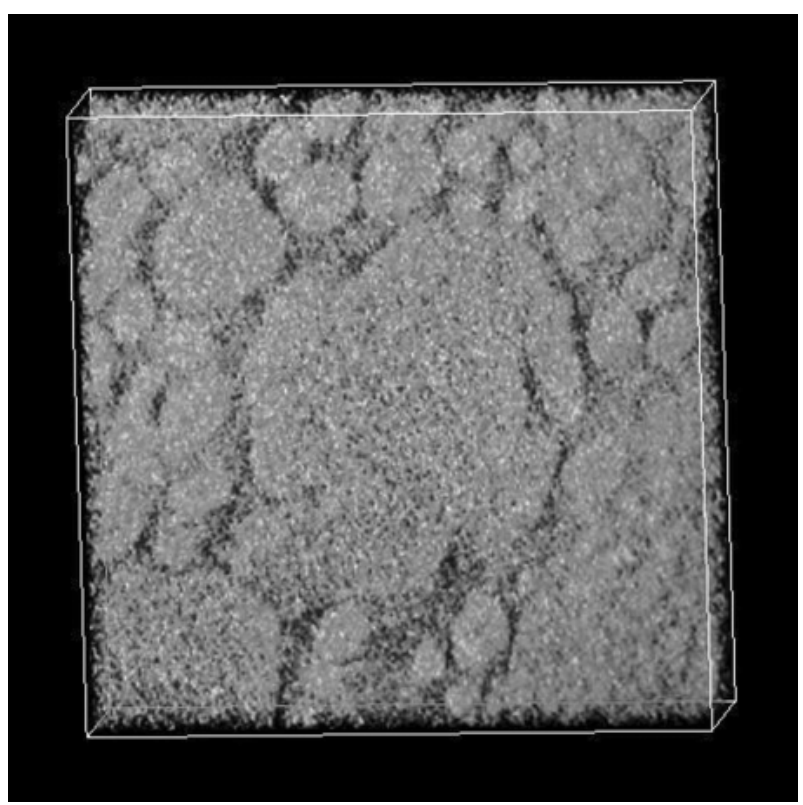

Fig. 6. Three-dimensional reconstructions of the rugged collagen fibril used Fig. 5 A-I with TEM Tomograph. A central large circle and several small oval zones can be clearly seen. And it seems to indicate that several thin fibrils join a central large fibril.

It is thought that biomechanical properties of the equine SDFT are established by the age of two years and are maintained for life [12]. Furthermore, it appears that the number of small collagen fibrils in the tendon increases with

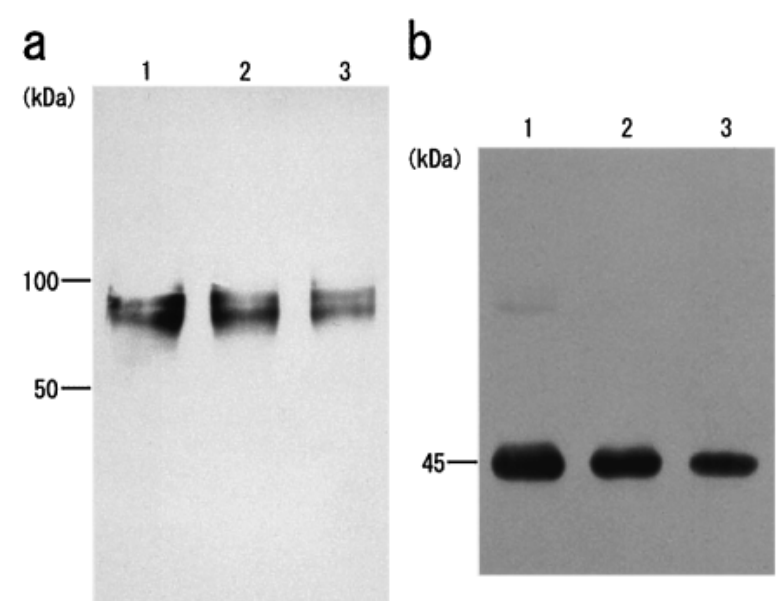

Fig. 7. a: Western blotting of decorin. Molecular sizes of decorin migrated slightly higher from the $\mathrm{pM}$ to the $\mathrm{dM}$ region. b: Western blotting of DS-depleted core protein of decorin. The amount of decorin decreases toward the $\mathrm{dM}$ region. Lane 1: pM region; Lane 2: mM region; Lane 3: dM region.

advance of age and increase in exercise $[2,8,18,20]$. However, different regions of the equine SDFT after maturation were not examined in those studies. Since horses of mature SDFT were used in the present work, changes in size and distribution of collagen fibrils would be explained some biomechanical properties of the tendon. It is interesting that the MAD increases in the direction of the $\mathrm{dM}$ region, while the number of small collagen fibrils gradually decreases. The diameters of collagen fibrils in the $\mathrm{pM}$ and $\mathrm{dM}$ regions were different. These findings suggest that the number of small collagen fibrils in the metacarpal zone of the equine SDFT decreases in the direction of the $\mathrm{dM}$ region after maturation. Actually, fibrils with large diameters $(>200 \mathrm{~nm})$ have greater tensile strength than do those with small diameters $(<40 \mathrm{~nm})$, corresponding to the number of lateral crosslinks. On the other hand, fibrils with small diameters contribute more than those having large diameters to elasticity by inhibition of fibril sliding or creeping, tendon deformation and collagen/non-collagen matrix interaction $[16,18$, 19]. These findings suggest that different regional changes of collagen fibril distribution would bring about different properties of the tendon in each region.

Although a difference was not found between fibril distributions in the $\mathrm{mM}$ and $\mathrm{dM}$ regions, many rugged fibrils in the $\mathrm{mM}$ region were observed. It has been reported that irregularly shaped fibrils are evidence of lateral association or fusion of collagen fibrils [6]. The same characteristics were also found in the $\mathrm{mM}$ region presumably from their morphological similarity of collagen fibrils. Three-dimensional graphics reconstructed from high-voltage electron microsocopic images proved that each rugged fibril with a large diameter fused with surrounding small fibrils. Accordingly, while the number of small fibrils decreased, the number of large collagen fibrils increased in the direc- 
tion of the $\mathrm{dM}$ region. This fusion is thought to be a mechanism of the formation of large fibrils in the $\mathrm{mM}$ region. Ramification and assembly of collagen bundles to form a network have been observed under a high-voltage electron microscope [4]. The present study showed that collagen fibrils form ramification by themselves fusion.

The major proteoglycan in the tendon is decorin, which anchors to d-bands with several collagen fibrils by noncovalent binding [21]. Decorin is composed of dermatan sulfate, which is a glycosaminoglycan that affects the morphology and formation of collagen fibrils and core protein by covalent binding [11]. Interfibrillar spaces between collagen fibrils and promotion of longitudinal growth of collagen fibrils have been shown to be influenced by decorin DS $[13,15,23]$. It has been reported that decorin changes length of decorin DS chains then controls interfibrillar space under some pathologic and developmental conditions [10, $14,15]$. Accordingly, important role of decorin in fusion and strength of collagen fibrils through this bondage would be undeniable [21, 22]. Activity inhibiting fibril segment association of decorin would also affect fibril growth [6]. Tendons of animals lacking decorin have been found to contain collagen fibrils with irregular diameters [9]. Down-regulation of decorin has also been shown to be correlated with the development of collagen fibrils with large diameters [17]. The increase in molecular size of decorin in the downward direction found in the present study indicates elongation of decorin DS chains and enlargement of interfibrillar spaces toward the dM region. This statement is supported by the finding of a decrease in CFI toward the $\mathrm{dM}$ region. The decrease in the amount but increase in molecular size of decorin found in Western blot analysis in accordance with increase in the number of collagen fibrils with large diameters toward the $\mathrm{dM}$ region supports the observation of lateral fusion in the $\mathrm{mM}$ region in $3 \mathrm{D}$ images. The amount of decorin in the $\mathrm{dM}$ region was decreased compared with that in the $\mathrm{mM}$ region. But rugged collagen fibrils were identified currently in the $\mathrm{mM}$ region. The interfibrillar space in the $\mathrm{dM}$ region with long decorin DS chaines was surmised that it was too large for such lateral fusion to occur. Although only effects of DS proteoglycans were found, effects of other leucine-rich proteoglycans such as biglycan was also expected in further study.

In this study, we have revealed that the distribution and diameter of collagen fibrils in the equine SDFT differ in some metacarpal regions. The amount and size of decorin also influence such alteration.

ACKNOWLEDGEMENT. This work was supported by the Sasakawa Scientific Research Grant from The Japan Science Society.

\section{REFERENCES}

1. Adachi, E., Hayashi, T. and Hashimoto, P. H. 1989. Immunoelectron microscopical evidence that type $\mathrm{V}$ collagen is a fibrillar collagen: importance for an aggregating capability of the preparation for reconstituting banding fibrils. Matrix 9: 232-
237.

2. Birch, H. L., Bailey, J. V., Bailey, A. J. and Goodship, A. E. 1999. Age-related changes to the molecular and cellular components of equine flexor tendons. Equine Vet. J. 31: 391-396.

3. Birk, D., Cohen, R., Geiger, B., Goodenough, D., Gumbiner, B., Hynes, R., Reichaedt, L., Ruoslahti, E., Trestad, R., Walsh, F. and Yurchenco, P. 1994. Cell junction, cell-cell adherens junction and extracellular matrix; The extracellular matrix of animals. pp. 971-995. In: Molecular Biology of the Cell. 3rd ed. (Alberts, B., Bray, D., Lewis, J., Raff, M., Roberts, K. and Watson, J. D. eds.), Garland Publishing, Inc., New York.

4. Birk, D. E. and Trelstad, R. L. 1989. Collagen fibril bundles: a branching assembly unit in tendon morphogenesis. Development 107: 437-443.

5. Birk, D. E., Fitch, J. M., Babiarz, J. P., Doane, K. J. and Linsenmayer, T. F. 1990. Collagen fibrillogenesis in vitro: interaction of types I and V collagen regulates fibril diameter. J. Cell Sci. 95: 649-657.

6. Birk, D. E., Nurminskaya, M. V. and Zycband, E. I. 1995. Collagen fibrillogenesis in situ: fibril segments undergo post-depositional modifications resulting in linear and lateral growth during matrix development. Dev. Dyn. 202: 229-243.

7. Canty, E. G. and Kadler, K. E. 2002. Collagen fibril biosynthesis in tendon: a review and recent insights. Comp. Biochem. Physiol. A Mol. Integr. Physiol. 133: 979-985.

8. Cherdchutham, W., Becker, C. K., Spek, E. R., Voorhout, W. F. and van Weeren, P. R. 2001. Effects of exercise on the diameter of collagen fibrils in the central core and periphery of the superficial digital flexor tendon in foals. Am. J. Vet. Res. 62: $1563-1570$.

9. Danielson, K. G., Baribault, H., Holmes, D. F., Graham, H., Kadler, K. E. and Iozzo, R. V. 1997. Targeted disruption of decorin leads to abnormal collagen fibril morphology and skin fragility. J. Cell Biol. 136: 729-743.

10. Derwin, K. A., Soslowsky, L. J., Kimura, J. H. and Plaas, A. H. 2001. Proteoglycans and glycosaminoglycan fine structure in the mouse tail tendon fascicle. J. Orthop. Res. 19: 269-277.

11. Flint, M. H., Craig, A. S., Reilly, H. C., Gillard, G. C. and Parry, D. A. 1984. Collagen fibril diameters and glycosaminoglycan content of skins--indices of tissue maturity and function. Connect. Tissue Res. 13: 69-81.

12. Gillis, C., Pool, R. R., Meagher, D. M., Stover, S. M., Reiser, K. and Willits, N. 1997. Effect of maturation and aging on the histomorphometric and biochemical characteristics of equine superficial digital flexor tendon. Am. J. Vet. Res. 58: 425-430.

13. Graham, H. K., Holmes, D. F., Watson, R. B. and Kadler, K. E. 2000. Identification of collagen fibril fusion during vertebrate tendon morphogenesis. The process relies on unipolar fibrils and is regulated by collagen-proteoglycan interaction. J. Mol. Biol. 295: 891-902.

14. Kuwaba, K., Kobayashi, M., Nomura, Y., Irie, S. and Koyama, Y. 2001. Elongated dermatan sulphate in post-inflammatory healing skin distributes among collagen fibrils separated by enlarged interfibrillar gaps. Biochem. J. 358: 157-163.

15. Kuwaba, K., Kobayashi, M., Nomura, Y., Irie, S. and Koyama, Y. 2002. Size control of decorin dermatan sulfate during remodeling of collagen fibrils in healing skin. J. Dermatol. Sci. 29: $185-194$.

16. Magnusson, S. P., Hansen, P. and Kjaer, M. 2003. Tendon properties in relation to muscular activity and physical training. Scand. J. Med. Sci. Sports 13: 211-223.

17. Nakamura, N., Hart, D. A., Boorman, R. S., Kaneda, Y., Shrive, N. G., Marchuk, L. L., Shino, K., Ochi, T. and Frank, 
C. B. 2000. Decorin antisense gene therapy improves functional healing of early rabbit ligament scar with enhanced collagen fibrillogenesis in vivo. J. Orthop. Res. 18: 517-523.

18. Parry, D. A., Barnes, G. R. and Craig, A. S. 1978. A comparison of the size distribution of collagen fibrils in connective tissues as a function of age and a possible relation between fibril size distribution and mechanical properties. Proc. R. Soc. Lond. B Biol. Sci. 203: 305-321.

19. Parry, D. A., Craig, A. S. and Barnes, G. R. 1978. Tendon and ligament from the horse: an ultrastructural study of collagen fibrils and elastic fibres as a function of age. Proc. R. Soc. Lond. B Biol. Sci. 203: 293-303.

20. Patterson-Kane, J. C., Wilson, A. M., Firth, E. C., Parry, D. A. and Goodship, A. E. 1997. Comparison of collagen fibril populations in the superficial digital flexor tendons of exercised and nonexercised thoroughbreds. Equine Vet. J. 29: 121-125.
21. Redaelli, A., Vesentini, S., Soncini, M., Vena, P., Mantero, S. and Montevecchi, F. M. 2003. Possible role of decorin glycosaminoglycans in fibril to fibril force transfer in relative mature tendons: a computational study from molecular to microstructural level. J. Biomech. 36: 1555-1569.

22. Scott, J. E. 1984. The periphery of the developing collagen fibril. Quantitative relationships with dermatan sulphate and other surface-associated species. Biochem. J. 218: 229-233.

23. Scott, J. E. 1993. Proteoglycans-fibrillar collagen interactions in tissue pp. 165-181. In: Dermatan Sulfate Proteoglycan as a Tissue Organizer (Scott, J. E. ed.), Portland Press, London.

24. Scott, J. E. 1995. Extracellular matrix, supramolecular organisation and shape. J. Anat. 187: 259-269.

25. Silver, F. H., Freeman, J. W. and Seehra, G. P. 2003. Collagen self-assembly and the development of tendon mechanical properties. J. Biomech. 36: 1529-1553. 